\title{
Charm hadroproduction in the atmosphere, QCD and neutrino astronomy
}

\author{
M. Benzke, M.V. Garzelli, B. Kniehl, G. Kramer, S. Moch*, G. Sigl \\ University of Hamburg, II Institute for Theoretical Physics \\ E-mail: sven-olaf.moch@desy.de
}

Production of charm in the atmosphere is one of the open problems at the interface between QCD and neutrino astronomy. In this contribution we provide an overview of the status of theoretical predictions, we discuss the role of the LHCb experiment in constraining cross-sections for the hadroproduction of charmed hadrons, and we compare the neutrino flux resulting from prompt decays of charmed mesons and baryons to the limits derived from experimental observations of leptons at IceCube.

Loops and Legs in Quantum Field Theory

24-29 April 2016

Leipzig, Germany

${ }^{*}$ Speaker. 
Precise QCD predictions are of interest not only for collider experiments, but even for highenergy astroparticle physics. Cosmic Rays (CR) accelerated in the Universe up to energies well above the maximum collision energy of the present most powerful collider, the Large Hadron Collider (LHC), may reach the Earth and produce ultra-high-energy (UHE) collisions with nuclei in the Earth's atmosphere. Although many open questions still exist on the CR acceleration mechanisms, and on the CR composition, especially at the highest energies, the all-particle energy spectrum of CRs impinging into the Earth's atmosphere has been measured by multiple experiments and its main features (knee, second knee, ankle) have been clearly recognized. The experiment that has accumulated the largest statistics at UHEs, the Pierre Auger Observatory, has shown that this spectrum extends in a continuous way up to laboratory energies $E_{l a b} \sim 4 \cdot 10^{19} \mathrm{eV}$, and presents a suppression afterwards. At present it is unclear if this suppression is due to the Greisen-ZatsepinKuzmin cutoff or to some upper limit on the acceleration power of astrophysical sources. In any case, it should be noted that a laboratory energy around $E_{\text {lab }}=10^{19} \mathrm{eV}$ corresponds to a centerof-mass energy $\sqrt{s} \sim 137 \mathrm{TeV}$ for proton-proton collisions, well above the present LHC $\sqrt{s}=13$ $\mathrm{TeV}$.

On the one hand, this means that higher energy colliders, like the Future Circular Collider (FCC), if built, will produce useful input for understanding the interactions of UHE CRs and will definitely provide an important framework to test the Standard Model at UHEs [1]. On the other hand, at present, we can still make tentative predictions for hadronic collisions at the highest energies of interest for astrophysics, with the help of QCD evolution equations and of extrapolations of results obtained at lower energies. Additional issues to be considered are the non-perturbative aspects of the interactions, described by phenomenological models including parameters which are fixed with the help of experimental data.

The dependence of parton distribution functions (PDFs) on the Bjorken- $x$ variable is one particular example of such non-perturbative aspects. Experimental data on deep-inelastic ep scattering collected at the HERA collider allowed to constrain PDFs down to $x \sim 10^{-4}$ and these data are taken into account in all modern PDF fits. On the other hand, Ref. [4] has recently proposed the usage of LHC data on charm and bottom hadroproduction at mid and forward rapidities to constrain PDFs even at smaller $x$. The idea has been exploited in new PDF fits extending down to $x \sim 5 \cdot 10^{-5}$ thanks to $\mathrm{LHCb}$ data collected at $\sqrt{s}=7 \mathrm{TeV}$ [2] in the rapidity range $2<y<4.5$. More recent data at $\sqrt{s}=13 \mathrm{TeV}$ [3] allow to extend the $x$ range even further, down to $x \sim 2 \cdot 10^{-6}$. The new PDF fits $[4,5]$ can be compared to the older pre-LHCb PDF fits. While in some cases (e.g. that of NNPDF 3.0n lo PDFs), it is evident that the inclusion of LHCb data put important constraints on a region where previous PDF uncertainties were simply huge, in some other cases, like that of the ABMIInlo fit [6], it turns out that including LHCb data or not does not make such an important difference. In fact, the extrapolation of the pre-LHC ABMIInI 0 fit to $x$ values $<10^{-4}$ is compatible with the post-LHCb fit proposed by the PROSA collaboration in Ref. [4], and the uncertainty band built from the $A B M 11$ nlo eigenvectors turns out to lie within the global uncertainty band corresponding to the PROSA eigenvectors, including fit, model and parameterization uncertainties. This is related to the fact that the ABM $1 \mathrm{n} l \circ$ fit allows already, without any a-posteriori change, a reasonable description of $\mathrm{LHCb}$ charm data, notwithstanding the fact that the fit was performed well before these data appear in the literature. As an illustration of this fact, the comparison of predictions on the hadroproduction of $D^{ \pm}$mesons with experimental data at $\sqrt{s}=7$ and $13 \mathrm{TeV}$, is 

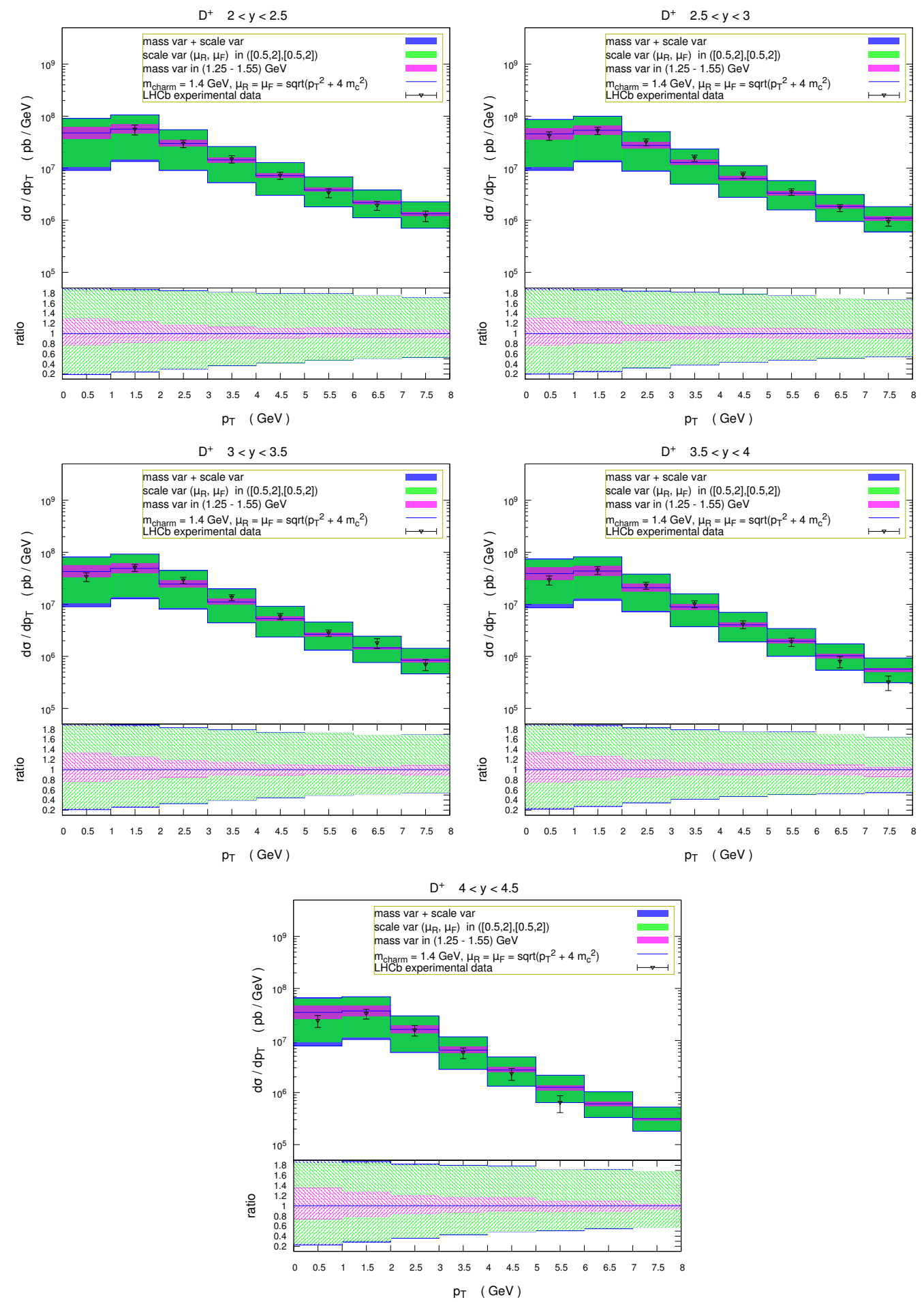

Figure 1: Differential distributions of the transverse momentum of $D^{ \pm}$mesons in $p p$ collisions at $\sqrt{s}=$ $7 \mathrm{TeV}$ computed by POWHEGBOX + PYTHIA 6 vs LHCb experimental data of Ref. [2]. Each panel refers to a different rapidity interval. Central theory predictions were obtained using the ABM $11 \mathrm{n} l o$ central PDF set, $\mu_{R}=\mu_{F}=\sqrt{p_{T, c}^{2}+4 m_{c}^{2}}$ and $m_{c}=1.4 \mathrm{GeV}$. Violet and green band refer to uncertainties due to charm mass and scale variation, respectively, computed as explained in the text and summed in quadrature in the blue band. In the lower inset of each panel the ratio of theoretical uncertainties with respect to the central theoretical prediction is shown. 

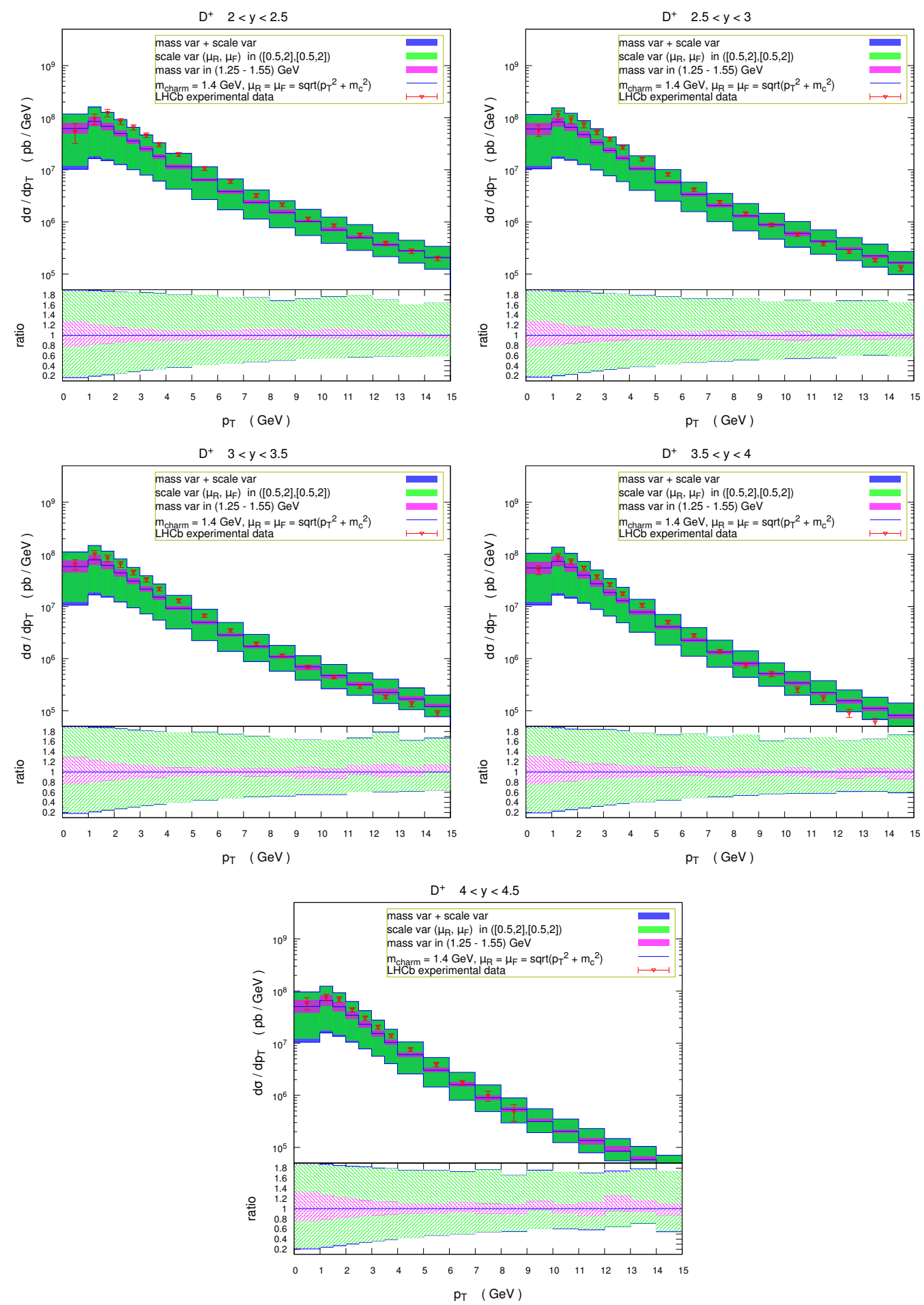

Figure 2: Differential distributions of the transverse momentum of $D^{ \pm}$mesons in $p p$ collisions at $\sqrt{s}=13$ $\mathrm{TeV}$ vs LHCb experimental data of Ref. [3]. Each panel refers to a different rapidity interval. Central theory predictions were obtained using the ABM1 $1 \mathrm{n}$ lo central PDF set, $\mu_{R}=\mu_{F}=\sqrt{p_{T, c}^{2}+4 m_{c}^{2}}$ and $m_{c}=1.4 \mathrm{GeV}$. Violet and green band refer to uncertainties due to charm mass and scale variation, respectively, summed in quadrature in the blue band, respectively, computed as explained in the text and summed in quadrature in the blue band. In the lower inset of each panel the ratio of theoretical uncertainties with respect to the central theoretical prediction is shown. 
shown in Fig. 1 and 2, respectively. Theoretical predictions include NLO QCD corrections matched to parton shower and hadronization, as available in POWHEGBOX [7] + PYTHIA 6 [8], use of the ABMI Inlo central PDF set, the on-shell charm mass fixed to $m_{c}=1.4 \pm 0.15 \mathrm{GeV}$ and renormalization and factorization scales centered at $\mu_{R}=\mu_{F}=\mu_{0}=\sqrt{p_{T}^{2}+4 m_{c}^{2}}$ and varied according to the combinations $\left(\mu_{R}, \mu_{F}\right) \in\{(0.5,0,5),(2,2),(0.5,1),(1,0.5),(1,2),(2,1)\} \mu_{0}$, of which we make the envelope. It is evident from Fig. 1 and 2 that the experimental uncertainties are smaller than the theoretical ones in almost all bins and that the agreement between theory predictions and experimental data seems to become slightly worse when going from $\sqrt{s}=7 \mathrm{TeV}$ to $\sqrt{s}=13 \mathrm{TeV}$, especially in the lowest rapidity bin $(2<y<2.5)$. This feature persists also for different PDFs (e.g. CT14nlo [9]), and even when using an approach based on the convolution of NLO fragmentation functions (FF) with the hard-scattering partonic cross-section including NLO QCD corrections, instead of our Monte Carlo procedure including hard-scattering matched to parton shower plus hadronization.

While the LHCb data explore the rapidity range $2<y<4.5$, the interaction of UHE CRs with the Earth's atmosphere gives rise to charmed mesons and baryons in an even larger rapidity range including more forward production. The LHCf experiment is able to explore forward pseudorapidities $(\eta>8.5)$, but is unable to detect charged charm particles. So the situation is not completely satisfactory both from the theoretical point of view, because of the large uncertainties affecting QCD predictions, and from the experimental point of view, because of the lack of data to crosscheck the validity of the theory in the forward region. Yet, one may provide predictions for charm in the atmosphere using the same tools as we have used for producing predictions for LHCb. This has been done in Ref. [10], subsequently followed-up by Ref. [11], which has used a similar method, but different PDFs (i.e. the aforementioned modified version of the NNPDF $3.0 \mathrm{n} l \circ$ fit) and we emphasize that these computations include elements of both perturbative and non-perturbative QCD.

Assuming that the interaction of cosmic rays with nuclei in the atmosphere can be approximated by the superposition of nucleon-nucleon $(N-N)$ interactions, the distribution of main interest for the computation of neutrino fluxes coming from charm hadroproduction in the atmosphere is $\mathrm{d} \sigma / \mathrm{d} x_{E}$, with $x_{E}=E_{h, l a b} / E_{N, l a b}$, for all lowest lying charmed hadrons $h_{c}=\left(D^{ \pm}, D^{0}, \overline{D^{0}}, D_{s}^{ \pm}\right.$and $\Lambda_{c}^{ \pm}$). This has to be evaluated in the energy range $E_{N, l a b}=10^{2}-10^{10} \mathrm{GeV}$ for $N N$ collisions, in order to produce neutrino fluxes in the neutrino energy range up to $E_{v} \sim 10^{7}-10^{8} \mathrm{GeV}$. An example of this distribution and of the related scale and charm mass uncertainties in case of $D^{0}$ hadroproduction at $E_{N, l a b}=10^{7} \mathrm{GeV}$ is shown in Fig. 3. Distributions at different energies are characterized by a similar shape.

The aforementioned differential distribution, together with some astrophysical input (in particular the CR primary fluxes and the nucleon-air total inelastic cross-section), is then the essential ingredient to the calculation of so-called cascade equations for particle evolution through an air column of given depth in the Earth's atmosphere, yielding the prompt neutrino fluxes. With the help of the so-called $Z$-moments, e.g., $Z_{p h_{c}}$ for charm hadroproduction in the atmosphere, obtained from the differential distribution by an integration over the whole kinematically accessible range, this set of coupled differential equations admits an approximate solution, when those moments $Z_{p h_{c}}$ are combined together with the $Z$-moments for $h_{c}$ decays into neutrinos $Z_{h_{c} v}$, those for $h_{c}$ regeneration $Z_{h_{c} h_{c}}$ and those for nucleon regeneration $Z_{N N}$, see for instance Refs. [12, 13, 10]. 

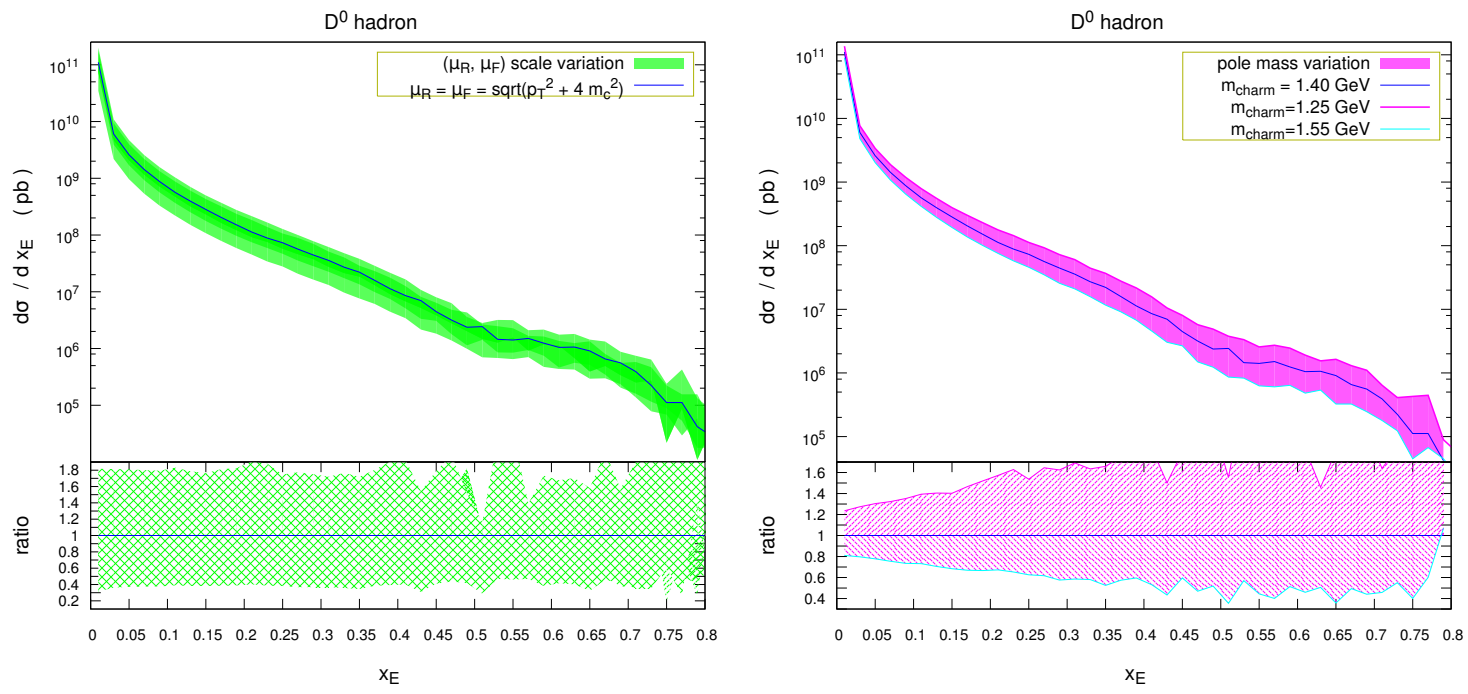

Figure 3: Differential distribution $d \sigma / d x_{E}$ for $N N \rightarrow c \bar{c} \rightarrow D^{0}+X$ computed with POWHEGBOX+PYTHIA at $E_{\text {lab }}=10^{7} \mathrm{GeV}$. Charm mass, scales and PDFs were chosen as in Fig. 1 and 2. The uncertainty bands related to $\left(\mu_{R}, \mu_{F}\right)$ scale and $m_{c}$ variation are shown in the left and right panels, respectively, whereas the ratio of the uncertainty bands with respect to the central prediction is depicted in the lower subpanels.
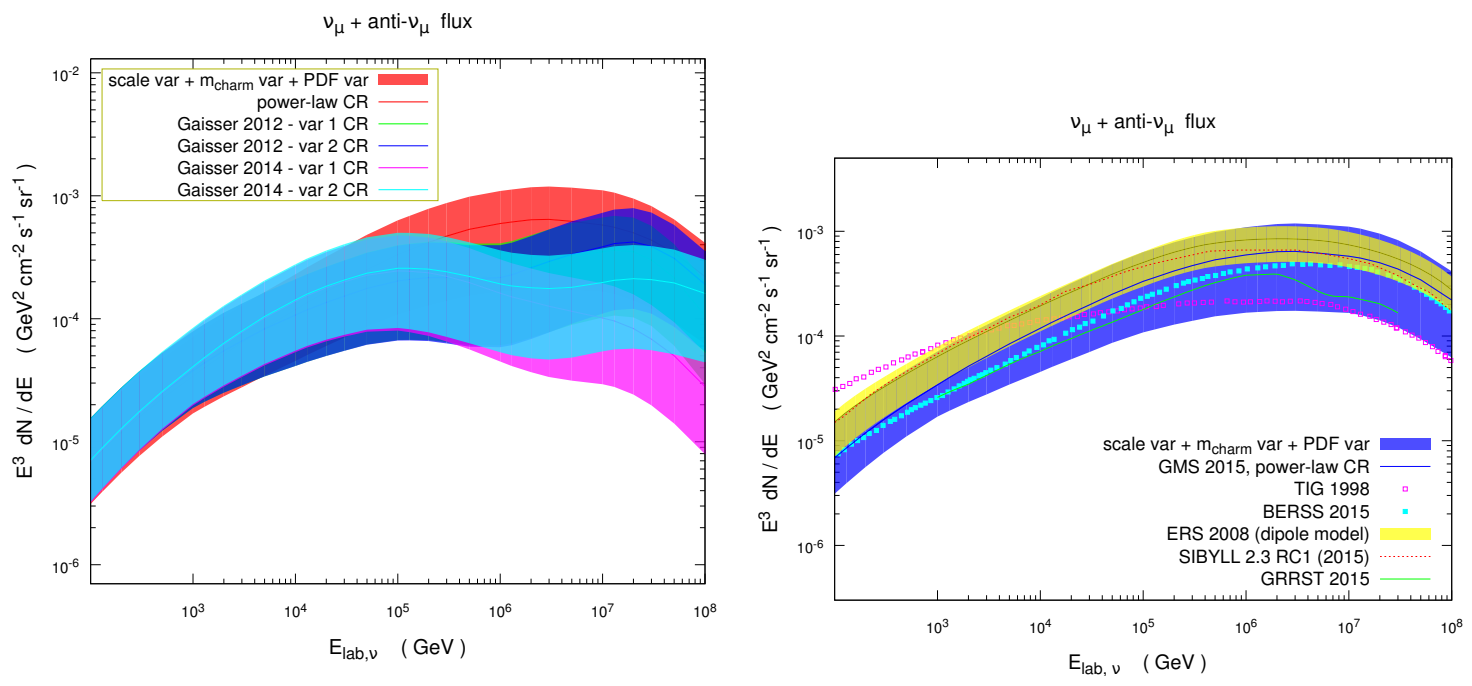

Figure 4: a) Our predictions for prompt neutrino fluxes (GMS 2015 [10]) and their uncertainties due to charm mass, scale and PDF variation, for five different primary CR all-nucleon spectra. b) Comparison with other predictions in the literature, all using the broken power-law CR spectrum. See text for more detail. 
Our final results for prompt neutrino fluxes are shown in Fig. 4.a, considering five different CR primary input spectra (the reference broken-power law spectrum used since several years in many computations of prompt neutrino fluxes, and four more realistic spectra which take into account recent experimental data on CR spectra) where the total uncertainty band on each flux comes from combining in quadrature uncertainties due to scale, charm mass and PDF variations. The comparison of our results with other predictions in the literature $[14,11,15,16,17]$ is shown in Fig. 4.b.

Our predictions presented so far were obtained from matrix elements and PDFs in the FixedFlavor-Number scheme (FFNS), where the charm quark is considered a massive parton. An alternative framework is represented by the variable-flavor-number scheme (VFNS) with zero-mass, where the charm quark is massless like the other light partons and may appear in the initial state. Its range of validity is in the high- $p_{T}$ region, where terms of the order $m_{c} / p_{T}$ can be neglected. A combination of both approaches is modeled by the general-mass VFNS (GM-VFNS). We have computed neutrino fluxes also using as a basis predictions for the hadroproduction of $h_{c}$ hadrons in the GM-VFNS, considering the implementation of Refs. $[18,19]$. This includes matrix elements for the hadroproduction of gluons, light and heavy quarks, complemented by fragmentation functions related to the probability that each of these elementary particles transform in a charmedhadron [20]. For scales above the charm mass threshold, the effects of the charm quark are included in the gluon splitting functions entering the standard QCD evolution equations for PDFs, as well as in the running of the strong coupling constant $\alpha_{S}$ with $\mu_{R}$. In analogy to Ref. [19] the renormalization scale of the computation is chosen as dynamical and its overall normalization is fixed such that predictions are extended to the whole $p_{T}$-region. In particular, we consider $\mu_{R}=\xi_{R} \sqrt{p_{T}^{2}+4 m_{h, c}^{2}}$, $\mu_{F}=\xi_{F} \sqrt{p_{T}^{2}+4 m_{h, c}^{2}}$ and we chose $\xi_{R}=1$ and $\xi_{F}=0.5$ after comparisons of the GM-VFNS predictions with the LHCb data on charm hadroproduction already discussed. CT14nlo PDFs were used throughout this calculation and the charm pole mass was fixed to a low value of $m_{c}=1.3 \mathrm{GeV}$ to have compatibility both with the PDF and FF charm mass thresholds. The comparison between our FFNS predictions with the central one obtained in the GM-VFNS computation, is shown for neutrino fluxes arising from the decay of different charmed hadrons in Fig. 5.a, and for the total flux in Fig. 5.b, where one can see that the GM-VFNS predictions turn out to lie inside the uncertainty band of the FFNS predictions for the entire interval of neutrino energies.

In the plot in Fig. 5.b also the present upper limit from the IceCube experiment on prompt neutrino flux is depicted, at $90 \%$ C.L. [21]. This limit is model dependent, i.e., it has been obtained by IceCube under the assumption that the prompt neutrino flux spectrum has a shape described by the so-called color dipole model used in Ref. [17]. As a matter of fact, IceCube has not yet observed a prompt atmospheric neutrino flux disentangled from the other flux components (conventional atmospheric flux and astrophysical flux), but the predictions produced so far by our and other groups seem to indicate that, with increasing statistics, the sensitivity for explicitly detecting prompt neutrinos will be reached soon. This is true provided the approximations used in the computation are reasonably valid. We note, that in particular the role of nuclear effects, instead of the superposition model, deserves indeed further exploration. Preliminary work in this direction has recently been started in Ref. [22], which might be subject to possible modifications, when the physics of nuclear PDFs and of interactions in a medium will be understood better. 

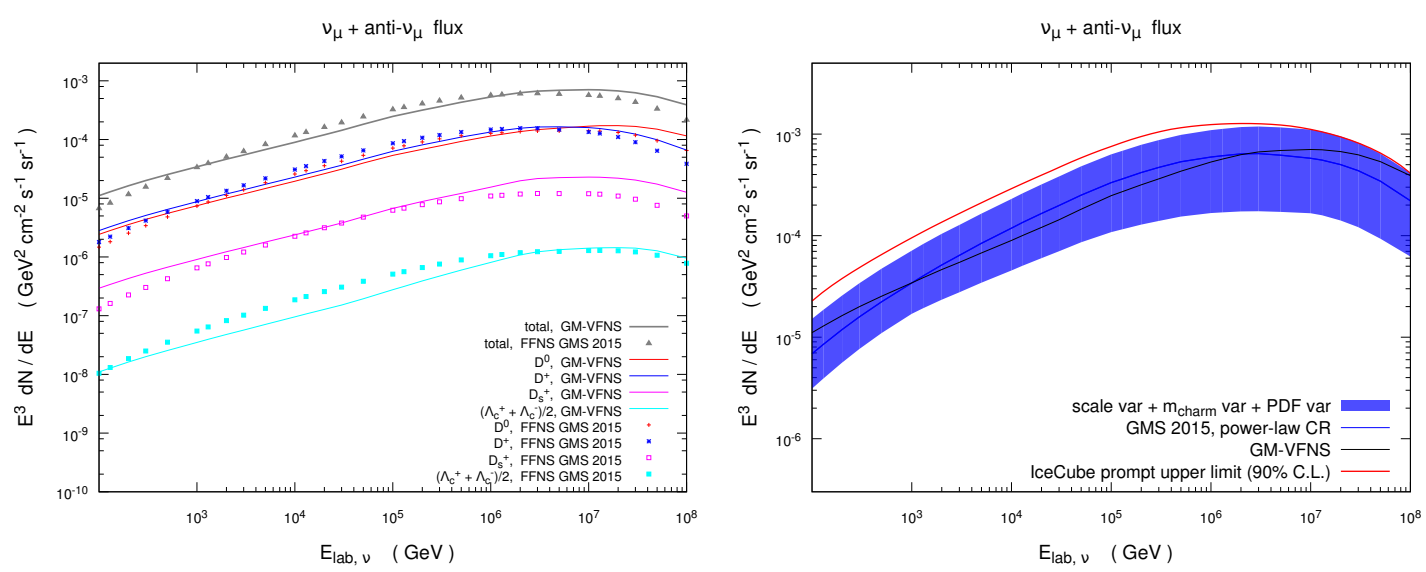

Figure 5: Comparison between theoretical predictions for the total $\left(v_{\mu}+\bar{v}_{\mu}\right)$ flux in the FFNS of Ref. [10] (GMS 2015) and those obtained in the GM-VFNS: a) separate central predictions related to neutrinos from different charmed hadrons, together with their sum; b) comparison between theoretical predictions for the total $\left(v_{\mu}+\bar{v}_{\mu}\right)$ flux in the FFNS, together with their uncertainty band (blue), and the central theoretical predictions obtained in the GM-VFNS (black). The present IceCube upper limit on prompt neutrino fluxes at $90 \%$ C.L. [21] is also shown (red line).

\section{Acknowledgments}

This work has been supported in part by Deutsche Forschungsgemeinschaft in Sonderforschungsbereich SFB 676 and by the European Commission through PITN-GA-2012-316704 (HIGGSTOOLS).

\section{References}

[1] M. L. Mangano et al., Physics at a $100 \mathrm{TeV}$ pp collider: Standard Model processes, arXiv:1607.01831.

[2] LHCb Collaboration, R. Aaij et al., Prompt charm production in pp collisions at sqrt(s)=7 TeV, Nucl. Phys. B871 (2013) 1-20, [arXiv:1302.2864].

[3] LHCb Collaboration, R. Aaij et al., Measurements of prompt charm production cross-sections in pp collisions at $\sqrt{s}=13 \mathrm{TeV}$, arXiv:1510.01707.

[4] PROSA Collaboration, O. Zenaiev et al., Impact of heavy-flavour production cross sections measured by the LHCb experiment on parton distribution functions at low x, Eur. Phys. J. C75 (2015), no. 8 396, [arXiv:1503.04581].

[5] R. Gauld, J. Rojo, L. Rottoli, and J. Talbert, Charm production in the forward region: constraints on the small-x gluon and backgrounds for neutrino astronomy, JHEP 11 (2015) 009, [arXiv:1506.08025].

[6] S. Alekhin, J. Blümlein, and S. Moch, Parton Distribution Functions and Benchmark Cross Sections at NNLO, Phys.Rev. D86 (2012) 054009, [arXiv: 1202.2281].

[7] S. Alioli, P. Nason, C. Oleari, and E. Re, A general framework for implementing NLO calculations in shower Monte Carlo programs: the POWHEG BOX, JHEP 1006 (2010) 043, [arXiv: 1002.2581 ]. 
[8] T. Sjostrand, S. Mrenna, and P. Z. Skands, PYTHIA 6.4 Physics and Manual, JHEP 0605 (2006) 026, [hep-ph/0603175].

[9] S. Dulat, T.-J. Hou, J. Gao, M. Guzzi, J. Huston, P. Nadolsky, J. Pumplin, C. Schmidt, D. Stump, and C. P. Yuan, New parton distribution functions from a global analysis of quantum chromodynamics, Phys. Rev. D93 (2016), no. 3 033006, [arXiv: 1506.07443 ].

[10] M. V. Garzelli, S. Moch, and G. Sigl, Lepton fluxes from atmospheric charm revisited, JHEP 10 (2015) 115, [arXiv:1507.01570].

[11] R. Gauld, J. Rojo, L. Rottoli, S. Sarkar, and J. Talbert, The prompt atmospheric neutrino flux in the light of LHCb, arXiv: 1511.06346.

[12] P. Lipari, Lepton spectra in the earth's atmosphere, Astropart.Phys. 1 (1993) 195-227.

[13] L. Pasquali, M. H. Reno, and I. Sarcevic, Lepton fluxes from atmospheric charm, Phys. Rev. D59 (1999) 034020, [hep-ph/9806428].

[14] A. Bhattacharya, R. Enberg, M. H. Reno, I. Sarcevic, and A. Stasto, Perturbative charm production and the prompt atmospheric neutrino flux in light of RHIC and LHC, arXiv:1502.01076.

[15] A. Fedynitch, R. Engel, T. K. Gaisser, F. Riehn, and T. Stanev, Calculation of conventional and prompt lepton fluxes at very high energy, EPJ Web Conf. 99 (2015) 08001, [arXiv: 1503.00544 ].

[16] P. Gondolo, G. Ingelman, and M. Thunman, Charm production and high-energy atmospheric muon and neutrino fluxes, Astropart. Phys. 5 (1996) 309-332, [hep-ph/ 9505417$].$

[17] R. Enberg, M. H. Reno, and I. Sarcevic, Prompt neutrino fluxes from atmospheric charm, Phys. Rev. D78 (2008) 043005, [arXiv: 0806.0418$].$

[18] B. A. Kniehl, G. Kramer, I. Schienbein, and H. Spiesberger, Inclusive Charmed-Meson Production at the CERN LHC, Eur. Phys. J. C72 (2012) 2082, [arXiv: 1202.0439 ].

[19] B. A. Kniehl, G. Kramer, I. Schienbein, and H. Spiesberger, Inclusive B-meson production at small $p_{T}$ in the general-mass variable-flavor-number scheme, Eur. Phys. J. C75 (2015), no. 3 140, [arXiv:1502.01001].

[20] T. Kneesch, B. A. Kniehl, G. Kramer, and I. Schienbein, Charmed-meson fragmentation functions with finite-mass corrections, Nucl. Phys. B799 (2008) 34-59, [arXiv: 0712.0481$].$

[21] IceCube Collaboration, L. Rädel and S. Schoenen, A measurement of the diffuse astrophysical muon neutrino flux using multiple years of IceCube data, PoS ICRC2015 (2015) 1079.

[22] A. Bhattacharya, R. Enberg, Y. S. Jeong, C. S. Kim, M. H. Reno, I. Sarcevic, and A. Stasto, Prompt atmospheric neutrino fluxes: perturbative QCD models and nuclear effects, arXiv:1607.00193. 